\title{
Serum IL-6 as a vital predictor of severe lung cancer
}

\author{
Jian $\mathrm{An}^{1,2,3} \wedge$, Qihua Gu ${ }^{1,2,3}$, Liming Cao ${ }^{1,2,3}$, Huaping Yang ${ }^{1,2,3}$, Pengbo Deng ${ }^{1,2,3}$, Chengping Hu ${ }^{1,2,3}$, \\ $\operatorname{Min} \operatorname{Li}^{1,2,3,4,5}$
}

${ }^{1}$ Department of Respiratory Medicine, National Key Clinical Specialty, Branch of National Clinical Research Center for Respiratory Disease, Xiangya Hospital, Central South University, Changsha, China; ${ }^{2}$ Xiangya Lung Cancer Center, Xiangya Hospital, Central South University, Changsha, China; ${ }^{3}$ Hunan Provincial Clinical Research Center for Respiratory Diseases, Changsha, China; ${ }^{4}$ Center for Molecular Medicine, Xiangya Hospital, Central South University, Changsha, China; ${ }^{5}$ Key Laboratory of Molecular Radiation Oncology of Hunan Province, Changsha, China Contributions: (I) Conception and design: M Li; (II) Administrative support: M Li; (III) Provision of study materials or patients: J An, Q Gu, L Cao, H Yang, P Deng, C Hu; (IV) Collection and assembly of data: J An; (V) Data analysis and interpretation: J An; (VI) Manuscript writing: All authors; (VII) Final approval of manuscript: All authors.

Correspondence to: Min Li. Department of Respiratory Medicine, National Key Clinical Specialty, Branch of National Clinical Research Center for Respiratory Disease, Xiangya Hospital, Central South University, Changsha, China. Email: limin2050@csu.edu.cn.

\begin{abstract}
Background Recent clinical studies have reported that some cytokines are associated with lung cancer prognosis and mortality. However, the relationship between cytokines and clinical outcomes in severe lung cancer patients was unclear. IL-6 as an important cytokine in inflammation, expression level in severe lung cancer patients was unknown.
\end{abstract}

Methods: A cohort of 55 severe lung cancer patients were enrolled retrospectively in this study. The clinical characteristics, including performance status (PS), therapeutic effect, and patients' adverse effects, were recorded. The association of cytokines and the concerned clinical outcomes were assessed by logistic regression analysis. The area under the curve (AUC) was assessed to evaluate the strength of prediction.

Results: The mean age of the patients was 59.8, and 42 patents were males. Increased IL-6 levels were associated with worse PS. Logistic regression analysis demonstrated that higher IL-6 was associated with an increased risk of progressive disease (PD) $(\mathrm{OR}=1.03$, 95\% CI: 1.0-1.06). The area under the ROC curve (AUC) of the model used for predicting PD was 0.821 .

Conclusions: Increased IL-6 levels are correlated with worse PS and are an essential predictor for PD in severe lung cancer patients. Monitoring the IL-6 level may represent an essential strategy in improving the prognosis of patients with severe lung cancer.

Keywords: Cytokines; severe lung cancer; IL-6, clinical outcomes

Submitted Sep 24, 2020. Accepted for publication Jan 08, 2021.

doi: 10.21037/apm-20-2229

View this article at: http://dx.doi.org/10.21037/apm-20-2229

\section{Introduction}

Lung cancer is one of the leading causes of cancer death (1) with a high overall death rate (1). The complicated tumor microenvironment in lung cancer, which consists of various immune cells and tumor cells, is widely considered as a vital factor in tumorigenesis and development $(2,3)$. Large amounts of inflammatory cells and pro-inflammatory factors cytokines (4), such as interleukin (IL), interferon (IFN), and tumor necrosis factor (TNF), play a crucial pro-inflammatory role in cancer progression. The tumor microenvironment provides a role in tumor progression which consist of vasculature, cancer-associated fibroblasts, immune cells, tumor-associated endothelial cells and extracellular matrix (5).

^ ORCID: 0000-0001-6410-1335. 
In the system, IL-6,VEGF were secreted to activate the major pathways on survival, migration and invasion through MAPK, PI3K and PKC.IL-6 regulates all hallmark including DNA damage repair, cell death, tissue invasion, metastasis and angiogenesis. Stromal cells and tumor cells interaction was enhanced by such soluble factors like IL-6 which was mainly secreted by tumor-associated macrophage (TAM) (6).

IL-6 is also a kind of inflammatory cytokine that control of acute inflammation. In cells express IL-6R, classical IL-6 signalling was activated. Free IL-6 binds soluble IL-6 receptor- $\alpha$ which will bind glycoprotein 130 complex and activates the anti-inflammatory pathways. Study discovered IL-6 expression in TAMs of the tumor stroma which is an independent prognostic factor for survival (7).

Specific cytokines may also serve as therapeutic targets of solid cancer (8), such as IL-2 in metastatic renal carcinoma $(9,10)$, and IFN- $\alpha$ in metastatic breast cancer (11) and renal cell carcinoma (12). Notably, a recent clinical trial study (13) demonstrated that IL- $1 \beta$ inhibitors were associated with lower incidence and lung cancer mortality. Some previous studies have demonstrated that the levels of IL-2 (14) and IL-6 (15) are associated with the prognosis of non-small cell lung cancer (NSCLC).

Cytokines, including IL-1, IL-6, and TNF-a (16) derived from a dysfunctional immune system or tumor is believed to play an essential role in cancer progression. Severe lung cancer here we defined as a specific syndrome of lung cancer patients with critical factors, including worse performance status (PS) scores of Eastern Cooperative Oncology Group (ECOG) (17), or patients with rapid disease progression after treatments, and patients who underwent severe adverse events. Performance status (PS) scores between 3 and 4 mean severe clinical symptoms caused by cancer (17). Previously study mentioned that IL-6 was a prognostic factor in lung cancer (18). However, the relationship between IL-6 in severe lung cancer patients has not been focused. In the present study, we aim to determine the cytokines level in severe lung cancer patients. We present the following article in accordance with the MDAR reporting checklist (available at http://dx.doi.org/10.21037/apm-20-2229).

\section{Methods}

\section{Patients and data collection}

We reviewed patients with a defined lung cancer diagnosis between January 2019 and August 2020 in Xiangya Hospital. We retrospectively enrolled severe lung cancer patients with critical factors, including lung cancer patients with worse ECOG PS scores, rapid disease progression after treatments, or patients who underwent severe adverse events. Patients with combining autoimmune disease or active infection were excluded. Finally, a cohort of 55 patients was included in the study. Demographics and clinical characteristics, including age, gender, pathology, and clinical stage, were collected. All procedures performed in this study involving human participants were in accordance with the Declaration of Helsinki (as revised in 2013). The study was approved by ethics committee of Xiangya Hospital. Individual consent for this retrospective analysis was waived.

\section{Cytokines detection}

The inflammation-associated cytokines were detected by enzyme-linked immunosorbent assay (ELISA) using fresh peripheral blood. The detections were performed following the chemiluminescence kit (SIEMENS, Siemens Healthcare Diagnostics Products Ltd.) specification, and the indexes were read on the IMMULITE 1000 Immunoassay System (SIEMENS, Immulite $\left.{ }^{\circledR} 1000\right)$. The cytokines and the reference ranges were as follows: IL-1 $1 \beta: 0-5 \mathrm{pg} / \mathrm{mL}, \mathrm{TNF}-\alpha$ : 0-8.1 pg/mL, IL-6: 0-5.9 pg/mL, IL-10: $11.4 \mathrm{pg} / \mathrm{mL}$. The Hospital laboratory detected ESR, CRP, PCT, and lymphocyte subsets.

\section{Clinical outcomes}

The patients' performance status (PS) was assessed by the Eastern Cooperative Oncology Group (ECOG) score, also named the WHO or Zubrod score. The scoring criteria divide patients' activity status into 6 levels ranging from 0 to 5: 0 , asymptomatic; 1 , symptomatic but completely ambulatory; 2 , symptomatic, and $<50 \%$ in bed during the day; 3 , symptomatic and $>50 \%$ in bed; 4 , bedbound; and 5, death. The therapeutic effect was divided into four groups: complete release (CR), partial release (PR), stable disease (SD), and progression of the disease (PD), according to Response Evaluation Criteria in Solid Tumors (RECIST) 1.1. Three to four-degree therapyrelated adverse effects were obtained, including myositis, hypotension, erosive gastritis, granulocytopenia, severe anemia, and gastrointestinal bleeding.

\section{Statistical analysis}

Descriptive analyses were performed with either means \pm standard deviation (continuous variables) or numbers/ 
Table 1 The demographics and clinical characteristics of patients with severe lung cancer in this study

\begin{tabular}{lc}
\hline Characteristic & Overall $(\mathrm{n}=55)$ \\
\hline Age $(\mathrm{y})$ & $59.8 \pm 8.7$ \\
Gender, $\mathrm{n}(\%)$ & \\
Male & $42(80.8)$ \\
Female & $13(19.2)$ \\
Pathology, $\mathrm{n}(\%)$ & \\
Adenocarcinoma & $25(45.5)$ \\
Squamous cell carcinoma & $23(41.8)$ \\
Small cell lung cancer & $5(9.1)$ \\
Others & $2(3.6)$ \\
Staging, $\mathrm{n}(\%)$ & \\
IIB & $1(1.8)$ \\
IIIA & $4(7.3)$ \\
IIIB & $5(9.1)$ \\
IIIC & $1(1.8)$ \\
IVA & $24(43.6)$ \\
IVB & $19(34.5)$ \\
IVC & $1(1.8)$ \\
\hline
\end{tabular}

percentages (categorical variables) to describe the patient's characteristics. The categorical variables were compared by Chi-square tests and continuous variables by ANOVA tests. The association of cytokines and the concerned clinical outcomes were assessed by logistic regression analysis. Odds ratios (OR) with $95 \%$ confidence intervals (CI) were calculated. The receiver operating characteristic (ROC) curve was calculated from the logistic regression model. The area under the curve (AUC) was assessed to evaluate the strength of prediction. A two-sided $\mathrm{P}$ value $<0.05$ was considered as statistically significant. All statistical analyses were performed using Stata software (version 16.0; StataCorp LLC, College Station, TX, USA).

\section{Results}

\section{Baseline characteristics of lung cancer patients}

The demographic and clinical characteristics of severe lung cancer patients enrolled in this study are present in Table 1 . The
Table 2 Association between cytokines and performance status

\begin{tabular}{|c|c|c|c|}
\hline \multirow{2}{*}{ ECOG score } & \multicolumn{2}{|c|}{ PS } & \multirow{2}{*}{$P$ value } \\
\hline & $0-2$ & $3-4$ & \\
\hline IL-6, n (\%) & & & 0.012 \\
\hline Normal & $35(71.4)$ & $10(28.6)$ & \\
\hline High & $14(40.0)$ & $15(60.0)$ & \\
\hline \multicolumn{4}{|l|}{ TNF- $\alpha, \mathrm{n}(\%)$} \\
\hline Normal & 48 (68.5) & 22 (31.5) & 0.109 \\
\hline High & $1(25.0)$ & $3(75.0)$ & \\
\hline \multicolumn{4}{|l|}{ IL-1 $\beta, \mathrm{n}(\%)$} \\
\hline Normal & $36(63.1)$ & $21(36.9)$ & 0.237 \\
\hline High & $13(76.4)$ & $4(23.6)$ & \\
\hline \multicolumn{4}{|c|}{ ESR/CRP/PCT, n (\%) } \\
\hline Normal & $23(67.6)$ & 11 (32.4) & 0.5 \\
\hline High & $26(65.0)$ & $14(35.0)$ & \\
\hline \multicolumn{4}{|l|}{$\mathrm{Th} / \mathrm{Ts}, \mathrm{n}(\%)$} \\
\hline Normal & 49 (72.0) & 19 (28.0) & 0.51 \\
\hline High & $4(66.7)$ & 2 (33.3) & \\
\hline
\end{tabular}

ECOG, Eastern Cooperative Oncology Group; PS, performance status; IL, interleukin; TNF- $\alpha$, tumor necrosis factor- $\alpha$; CD, leukocyte differentiation antigen; Ts, suppressor T-cell; Th, helper T-cell.

mean age was 59.8 years ranging from 38 to 74 , and $80.8 \%$ of the patients were male. The pathologic type was distributed as adenocarcinoma $45.5 \%$, squamous cell carcinoma $41.8 \%$, small cell lung cancer $9.1 \%$, and $3.6 \%$. The clinical-stage was distributed as $18.2 \%$ for stage III and $79.9 \%$ for stage IV. Of 74 cytokines expression tests, $39.1 \%$ (29/74) showed a high expression level of IL-6 and 5.4\% (4/74) with an elevated level of TNF- $\alpha$. The average levels of CRP, ESR, and PCT were $12.3 \mathrm{mg} / \mathrm{L}, 53.5 \mathrm{~mm} / \mathrm{h}, 0.1 \mathrm{ng} / \mathrm{mL}$, respectively.

\section{Association between cytokines and performance status}

The association of cytokines and PS is shown in Table 2. Patients with ECOG score 0-2, 35 (71.4\%) had normal expression of IL-6. In patients with ECOG score 3-4, 15 (60\%) patients had a high expression of IL-6 ( $\mathrm{P}=0.012)$. Other detected cytokines were not associated with PS. In patients with worse PS scores, only 3 in 22 patients had high TNF- $\alpha$ levels $(\mathrm{P}=0.1)$. Differences were not seen in IL-1 $\beta$, ESR/CRP/PCT, and Th/Ts $(\mathrm{P}=0.237 / 0.5 / 0.51)$. 
Table 3 Comparisons of the demographic, clinical characteristics, and cytokines, by different treatment effects

\begin{tabular}{|c|c|c|c|c|c|c|}
\hline Characteristic & $\mathrm{CR}(\mathrm{n}=2)$ & PR (n=23) & $S D(n=26)$ & $P D(n=23)$ & $\mathrm{P}$ & $\mathrm{P}$ trend \\
\hline Male & $1(50 \%)$ & $14(87.5 \%)$ & 13 (100\%) & 14 (66.7\%) & 0.058 & 0.293 \\
\hline Staging & & & & & $0.002^{*}$ & $0.001^{*}$ \\
\hline IIB & 0 & $1(5.6 \%)$ & 0 & 0 & & \\
\hline IIIB & 0 & $3(16.7 \%)$ & $1(7.1 \%)$ & $1(4.8 \%)$ & & \\
\hline IIIC & 0 & 0 & $1(7.1 \%)$ & 0 & & \\
\hline IVA & 0 & 8 (44.4\%) & 7 (50.0\%) & 9 (42.9\%) & & \\
\hline IVB & 0 & $4(22.2 \%)$ & 4 (28.6\%) & 11 (52.4\%) & & \\
\hline IL-6 & $3.1(2.1-4.0)$ & $2.7(2.0-4.6)$ & $9.0(3.5-18.8)$ & $9.5(5.0-27.0)$ & $0.001^{*}$ & $<0.001^{*}$ \\
\hline TNF- $\alpha$ & $6.3(5.0-7.6)$ & $9.3(6.0-11.3)$ & $9.7(6.9-12.3)$ & $9.2(7.0-13.0)$ & 0.421 & 0.141 \\
\hline IL-1 $\beta$ & $5.0(5.0-5.0)$ & $5.0(5.0-7.0)$ & $5.0(5.0-5.0)$ & $5.0(5.0-6.0)$ & 0.719 & 0.754 \\
\hline IL-10 & $5.0(5.0-5.0)$ & $5.0(5.0-5.0)$ & $5.0(5.0-5.0)$ & $5.0(5.0-5.0)$ & 0.535 & 0.186 \\
\hline CD3 & $73.3 \pm 9.5$ & $70.1 \pm 7.9$ & $68.0 \pm 12.7$ & $68.0 \pm 11.0$ & 0.819 & 0.459 \\
\hline CD3CD4\% & $44.9 \pm 3.0$ & $39.6 \pm 7.7$ & $38.1 \pm 9.7$ & $37.6 \pm 9.1$ & 0.648 & 0.170 \\
\hline CD3CD8\% & $26.4 \pm 5.2$ & $27.0 \pm 7.4$ & $26.6 \pm 8.6$ & $27.0 \pm 8.1$ & 0.996 & 0.921 \\
\hline $\mathrm{Th} / \mathrm{Ts}$ & $1.7(1.5-1.9)$ & $1.4(1.1-2.0)$ & $1.2(1.0-2.2)$ & $1.3(1.0-1.9)$ & 0.897 & 0.562 \\
\hline
\end{tabular}

*, IL-6 was higher in patients with SD and PD compared with CR and PR patients. P<0.001. IL, interleukin; TNF- $\alpha$, tumor necrosis factor- $\alpha$; CD, leukocyte differentiation antigen; Ts, suppressor T-cell; Th, helper T-cell; CR, complete response; PR, partial response; PD, progressive disease; SD, stable disease.

\section{Characteristics of study cohort by therapeutic effect classification}

The comparison of demographic, clinical characteristics, and cytokines, by different treatment effects, are shown in Table 3. The stage IVA and IVB of PD patients were $42.9 \%$ and $52.4 \%$, respectively. Patients with IIB and IIIA showed no progress. Most PR and SD patients were IV stage patients $(\mathrm{P}=0.002)$. The expression of IL-6 was higher in patients with $\mathrm{SD}$ and $\mathrm{PD}$ when compared to patients with $\mathrm{CR}$ and $\mathrm{PR}(\mathrm{P}=0.001)$. The difference was not observed in age, gender, metastasis status, and other cytokines.

\section{IL-6 as a predictor of progressive disease (PD) in severe lung cancer}

The logistic regression analysis results for the association of inflammatory cytokines and PD are presented in Table 4. Univariate analysis revealed that increasing IL-6 levels is associated with a higher risk of $\mathrm{PD}(\mathrm{OR}=1.03,95 \% \mathrm{CI}$ : 1.00-1.06), while other inflammatory cytokines were not associated with PD in severe lung cancer. The predictive performance of the IL- 6 was evaluated by ROC curve analysis, with a total of 74 evaluations. The area under the ROC curve (AUC) of the prediction model was 0.821 (Figure 1).

\section{Association of cytokines and therapy-related adverse effect}

The association of cytokines and the incidence of therapyrelated adverse effects were assessed (Table 5). The total adverse event incident rate was $15 \%$, and nine cases of SAE were observed, including two myositis, one hypotension, one erosive gastritis, three granulocytopenia, one severe 
Table 4 The association of inflammatory cytokines and the progression of the disease

\begin{tabular}{lccc}
\hline Variable & OR & $95 \% \mathrm{Cl}$ & P value \\
\hline CRP & 1.04 & $0.97-1.11$ & 0.276 \\
ESR & 1.02 & $0.99-1.04$ & 0.100 \\
IL-6 & 1.03 & $1.00-1.06$ & 0.039 \\
TNF- $\alpha$ & 1.04 & $0.06-1.13$ & 0.363 \\
IL-1 $\beta$ & 1.00 & $0.99-1.02$ & 0.530 \\
IL-10 & 1.05 & $0.94-1.16$ & 0.395 \\
CD3 & 0.99 & $0.95-1.02$ & 0.438 \\
CD3CD4\% & 0.975 & $0.930-1.020$ & 0.315 \\
CD3CD8\% & 1.000 & $0.959-1.050$ & 0.990 \\
Th/Ts & 0.940 & $0.567-1.557$ & 0.810 \\
\hline
\end{tabular}

IL, interleukin; TNF- $\alpha$, tumor necrosis factor- $\alpha$; CD, leukocyte differentiation antigen; Ts, suppressor T-cell; Th, helper T-cell; CRP, C-reactive protein; ESR, erythrocyte sedimentation rate.

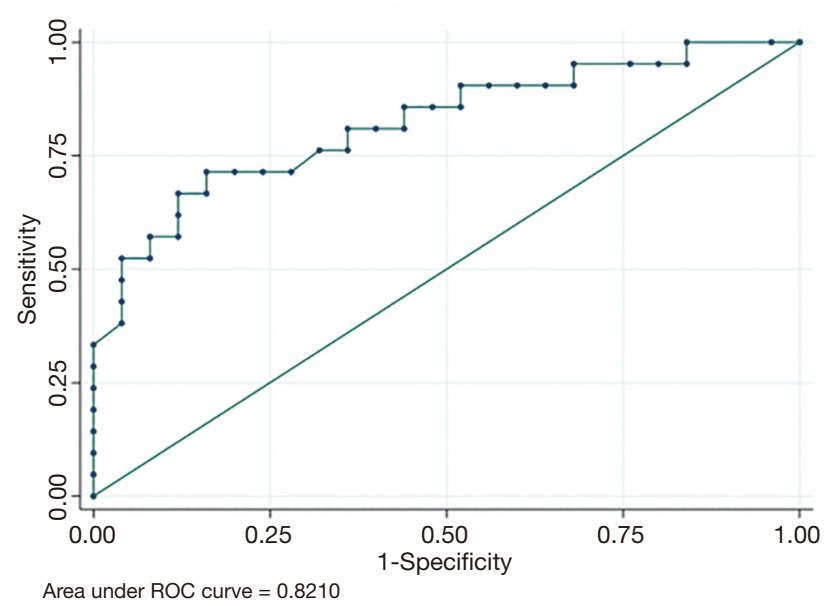

Figure 1 ROC curve for IL-6 to predict the progression of disease in severe lung cancer patients.

Table 5 Association of cytokines and therapy related adverse effect

\begin{tabular}{lccccccc}
\hline Adverse events & $\begin{array}{c}\text { Total } \\
(\mathrm{N}=74)\end{array}$ & $\begin{array}{c}\text { High TNF- } \alpha \\
(\mathrm{n}=30)\end{array}$ & $\begin{array}{c}\text { Normal TNF- } \alpha \\
(\mathrm{n}=44)\end{array}$ & $\begin{array}{c}\text { High IL-1 } \\
(\mathrm{n}=9)\end{array}$ & $\begin{array}{c}\text { High IL-1 } \\
(\mathrm{n}=65)\end{array}$ & $\begin{array}{c}\text { High IL-6 } \\
(\mathrm{n}=40)\end{array}$ & $\begin{array}{c}\text { Normal IL-6 } \\
(\mathrm{n}=34)\end{array}$ \\
\hline All events & $10(15 \%)$ & $7(23.3 \%)$ & $2(4.5 \%)$ & $2(22.2 \%)$ & $8(12.3 \%)$ & $6(15 \%)$ & $4(11.7 \%)$ \\
Myositis & 2 & 0 & 1 & 0 & 2 & 1 & 1 \\
Hypotension & 1 & 1 & 0 & 1 & 0 & 1 & 0 \\
Erosive gastritis & 1 & 1 & 0 & 0 & 1 & 0 & 1 \\
Granulocytopenia & 3 & 2 & 1 & 0 & 3 & 2 & 1 \\
Severe anemia & 1 & 1 & 0 & 0 & 1 & 0 & 1 \\
Gastrointestinal bleeding & 1 & 1 & 0 & 0 & 1 & 1 & 0 \\
Interstitial lung disease & 1 & 1 & 0 & 1 & 0 & 1 & 0 \\
\hline
\end{tabular}

anemia, and one gastrointestinal bleeding. The adverse event incident rates in high/normal TNF- $\alpha$ and IL-1 $\beta$ patients were $23.3 \% / 4.5 \%$ and $22.2 \% / 12.3 \%$, respectively; however, the rate of high/normal IL-6 patients was $15 \% / 11.7 \%$.

\section{Discussion}

There were a few markers which indicate the status of lung cancer patients. The balance between cancer cells and humoral/cellular immunity can be evaluated. Tocilizumab was reported to treat Cytokine Release Syndrome (CRS) with fevers and multiorgan dysfunction caused by cancer immunotherapies $(19,20)$. We noticed that some patients PS score deteriorate besides acute infection, most of them had high inflammatory status.

In the study, we evaluated whether cytokines are predictors of clinical outcomes in severe lung cancer patients. IL- $1 \beta$ was secreted by T lymphocyte, ESR/CRP/ PCT were usually correlated with infection of inflammation which might trigger signal activation. Th/Ts reflect the balance of cellular and humoral immunity. IL-6 is one of the most important cytokines. Elevated IL-6 was observed in patients with infective disease as SARS and COVID-19 were 
believed related to $\mathrm{T}$ lymphocyte hyperactivated leading to vascular dysfunction and pulmonary damage (19). Several studies have reported the predictive value of IL-6 for the prognosis of different cancers. Lee et al. reported that IL-6 was a prognostic indicator for hepatocellular carcinoma in patients treated with transarterial chemoembolization among cytokines (21). An experimental study found that overexpression of IL-6 in colon tumor cells significantly increased tumor growth in vivo of colon tumors (22). Osuala et al. found that IL-6 signaling between preinvasive ductal carcinoma in situ cells (DCIS) and stromal CAFs represent an important factor in the initiation of DCIS progression to invasive breast carcinoma (23). IL-6 also reportedly promoted metastasis of non-small-cell lung cancer by upregulating TIM-4 via NF- $\mathrm{KB}$ (24). Our study discovered that IL-6 serves as an important biomarker to predict severe lung cancer's poor outcome.

We identified that patients with high IL-6 were at high risk of worse PS and PD compared to those with normal levels of IL-6. In a previous study, IL-6 levels were deemed as a biomarker to assess the activity of irAEs (19); however, in our study, the predictive value of IL-6 in AEs of severe lung cancer was not obvious. The correlation between IL-6 and severe lung cancer indicates it may be a potential therapeutic target. Tocilizumab is a recombinant humanized monoclonal anti-IL-6R antibody. It binds both soluble and membrane-bound IL-6R to inhibit IL-6-mediated signaling. Tocilizumab has been approved by The US Food and Drug Administration for the treatment of severe CAR T-cell-induced CRS (20). If IL-6 was elevated in severe lung cancer with worse PS or rapid PD, we should evaluate its role in treatment decisions, particularly in anti-IL-6 treatment like Tocilizumab.

In our data, there were no significant difference in IL- $1 \beta$, $\mathrm{ESR} / \mathrm{CRP} / \mathrm{PCT}$, and $\mathrm{Th} / \mathrm{Ts}$ in different performance status. The study revealed that TNF-a, IL-1 $\beta$, IL-10, lymphocyte subgroup had no relation with therapeutic effect.

Besides IL-6, other cytokines were reported to be related to the prognosis of different cancers. Lima et al. enrolled 74 patients (weight stable cancer $n=31$ and cachectic cancer $\mathrm{n}=43$ ) diagnosed with colorectal cancer (CRC) and observed higher protein expression of inflammatory cytokines and growth factors in the tumor and serum of cachectic cancer patients when compared to weight-stable counterparts, such as epidermal growth factor, granulocyte-macrophage colony-stimulating factor, interferon- $\alpha$, and interleukin (IL)-8 (25). Camargo et al. reported that NF- $\kappa$ Bp65 and its target genes expression (TNF- $\alpha$, IL- $1 \beta$,

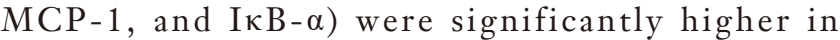
cachectic cancer patients, which proved the function of white adipose tissue in inflammation (26). Martins et al. found that an increased level of IL-4 was associated with longer survival time in females with mammary tumors (27). IL-38 is a reliable and sensitive biomarker for distinguishing between CRC and non-cancer colonic tissue. There is a positive correlation between colonic IL-38 in CRC prognosis, particularly in advanced CRC. This supports IL-38 as a reliable and consistent independent factor in predicting CRC prognosis (28). However, we found no significant association between other cytokines and clinical outcomes, including TNF- $\alpha$, IL-1 $\beta$, and IL-10. Additional cytokines should be studied in the future.

In conclusion, we determined that in severe lung cancer patients, a high level of IL-6 was associated with worse PS and PD. Also, IL-6 may act as an important predictor of PD in severe lung cancer patients. IL-6 is relatively simple to detect and interpret, and monitoring the IL-6 level may represent an important strategy to improve the prognosis of patients with severe lung cancer.

\section{Acknowledgments}

Funding: This investigation was supported by the National Multidisciplinary Cooperative Diagnosis and Treatment Capacity Building Project for Major Diseases (Lung Cancer), Natural Science Foundation of China (81903020), and China Postdoctoral Science Foundation (2019M652812).

\section{Footnote}

Reporting Checklist: The authors have completed the MDAR checklist. Available at http://dx.doi.org/10.21037/apm20-2229

Data Sharing Statement: Available at http://dx.doi. org/10.21037/apm-20-2229

Conflicts of Interest: All authors have completed the ICMJE uniform disclosure form (available at http://dx.doi. org/10.21037/apm-20-2229). The authors have no conflicts of interest to declare

Etbical Statement: The authors are accountable for all aspects of the work in ensuring that questions related to the accuracy or integrity of any part of the work are 
appropriately investigated and resolved. All procedures performed in this study involving human participants were in accordance with the Declaration of Helsinki (as revised in 2013). The study was approved by ethics committee of Xiangya Hospital. Individual consent for this retrospective analysis was waived.

Open Access Statement: This is an Open Access article distributed in accordance with the Creative Commons Attribution-NonCommercial-NoDerivs 4.0 International License (CC BY-NC-ND 4.0), which permits the noncommercial replication and distribution of the article with the strict proviso that no changes or edits are made and the original work is properly cited (including links to both the formal publication through the relevant DOI and the license). See: https://creativecommons.org/licenses/by-nc-nd/4.0/.

\section{References}

1. Siegel RL, Miller KD, Jemal A. Cancer statistics, 2020. CA: Cancer J Clin 2020;70:7-30.

2. Quail DF, Joyce JA. Microenvironmental regulation of tumor progression and metastasis. Nat Med 2013; 19:1423-37.

3. Peng L, Wang Y, Fei S, et al. The effect of combining Endostar with radiotherapy on blood vessels, tumorassociated macrophages, and $\mathrm{T}$ cells in brain metastases of Lewis lung cancer. Transl Lung Cancer Res 2020;9:745-760.

4. Orditura M, Romano C, De Vita F, et al. Behaviour of interleukin-2 serum levels in advanced non-small-cell lung cancer patients: relationship with response to therapy and survival. Cancer Immunol Immunother 2000;49:530-6.

5. Wu T, Dai Y. Tumor microenvironment and therapeutic response. Cancer Lett 2017;387:61-8.

6. Nguyen DP, Li J, Tewari AK. Inflammation and prostate cancer: the role of interleukin 6. BJU Int 2014;113:986-92.

7. Unver N, McAllister F. IL-6 family cytokines: Key inflammatory mediators as biomarkers and potential therapeutic targets. Cytokine Growth Factor Rev 2018;41:10-17

8. Rosenberg SA, Lotze MT, Yang JC, et al. Experience with the use of high-dose interleukin-2 in the treatment of 652 cancer patients. Ann Surg 1989;210:474-84; discussion 484-5.

9. Fyfe G, Fisher RI, Rosenberg SA, et al. Results of treatment of 255 patients with metastatic renal cell carcinoma who received high-dose recombinant interleukin-2 therapy. J Clin Oncol 1995;13:688-96.

10. Gutterman JU, Blumenschein GR, Alexanian R, et al. Leukocyte interferon-induced tumor regression in human metastatic breast cancer, multiple myeloma, and malignant lymphoma. Ann Intern Med 1980;93:399-406.

11. Amato R. Modest effect of interferon alfa on metastatic renal cell carcinoma. Lancet 1999;353:6-7.

12. Ridker PM, MacFadyen JG, Thuren T, et al. Effect of interleukin- $1 \beta$ inhibition with canakinumab on incident lung cancer in patients with atherosclerosis: exploratory results from a randomised, double-blind, placebocontrolled trial. Lancet 2017;390:1833-42.

13. Lanser L, Kink P, Egger EM, et al. Inflammation-Induced Tryptophan Breakdown is Related With Anemia, Fatigue, and Depression in Cancer. Front Immunol 2020;11:249.

14. Songür N, Kuru B, Kalkan F, et al. Serum interleukin-6 levels correlate with malnutrition and survival in patients with advanced non-small cell lung cancer. Tumori 2004;90:196-200.

15. Waldmann TA. Cytokines in cancer immunotherapy. Cold Spring Harb Perspect Biol 2018;10:a028472.

16. Moses AG, Maingay J, Sangster K, et al. Proinflammatory cytokine release by peripheral blood mononuclear cells from patients with advanced pancreatic cancer: relationship to acute phase response and survival. Oncol Rep 2009;21:1091-5.

17. Kelly CM, Shahrokni A. Moving beyond Karnofsky and ECOG Performance Status Assessments with New Technologies. J Oncol 2016;2016:6186543.

18. Pei BX, Sun BS, Zhang ZF, et al. Interstitial tumorassociated macrophages combined with tumor-derived colony-stimulating factor- 1 and interleukin-6, a novel prognostic biomarker in non-small cell lung cancer. J Thorac Cardiovasc Surg 2014;148:1208-1216.e2.

19. Soy M, Keser G, Atagündüz P, et al. Cytokine storm in COVID-19: pathogenesis and overview of antiinflammatory agents used in treatment. Clin Rheumatol 2020;39:2085-94.

20. Fishman JA, Hogan JI, Maus MV. Inflammatory and Infectious Syndromes Associated With Cancer Immunotherapies. Clin Infect Dis 2019;69:909-20.

21. Lee HL, Jang JW, Lee SW, et al. Inflammatory cytokines and change of Th1/Th2 balance as prognostic indicators for hepatocellular carcinoma in patients treated with transarterial chemoembolization. Sci Rep 2019;9:3260.

22. Liang B, Li L, Miao R, et al. Expression of Interleukin-6 and Integrin-6 in Colon Cancer: Association with Clinical Outcomes and Prognostic Implications. Cancer Invest 
2019;37:174-84.

23. Osuala KO, Sameni M, Shah S, et al. Il-6 signaling between ductal carcinoma in situ cells and carcinomaassociated fibroblasts mediates tumor cell growth and migration. BMC Cancer 2015;15:584.

24. Ke W, Zhang L, Dai Y. The role of IL-6 in immunotherapy of non-small cell lung cancer (NSCLC) with immune-related adverse events (irAEs). Thorac Cancer 2020;11:835-9.

25. Lima JDCC, Simoes E, de Castro G, et al. Tumourderived transforming growth factor- $\beta$ signalling contributes to fibrosis in patients with cancer cachexia. J Cachexia Sarcopenia Muscle 2019;10:1045-59.

26. Camargo RG, Riccardi DM, Ribeiro HQ, et al. NF-кBp65 and Expression of Its Pro-Inflammatory Target Genes Are Upregulated in the Subcutaneous Adipose Tissue of Cachectic Cancer Patients. Nutrients 2015;7:4465-79.

27. Martins GR, Gelaleti GB, Moschetta MG, et al. Proinflammatory and Anti-Inflammatory Cytokines Mediated by NF- $\kappa$ B Factor as Prognostic Markers in Mammary Tumors. Mediators Inflamm 2016;2016:9512743.

28. Ibrahim ML, Lu C, Klement JD, Redd PS, et al. Expression profiles and function of IL6 in polymorphonuclear myeloid-derived suppressor cells. Cancer Immunol Immunother 2020;69:2233-45.

(English Language Editors: L. Gray and J. Chapnick)
Cite this article as: An J, Gu Q, Cao L, Yang H, Deng P, Hu C, Li M. Serum IL-6 as a vital predictor of severe lung cancer. Ann Palliat Med 2021;10(1):202-209. doi: 10.21037/apm-20-2229 